\title{
Qualidade dos serviços prestados em transporte rodoviário interestadual durante a pandemia da COVID-19
}

\author{
Quality of services provided in interestadual road transport during COVID-19 pandemic
}

Calidad de los servicios prestados en el transporte carretero interestatal a lo largo de la pandemia de COVID-19

\section{Resumo}

Este artigo apresenta uma aplicação da ferramenta SERVQUAL adaptada para avaliar a qualidade do serviço prestado em uma empresa de transporte rodoviário interestadual de passageiros no estado de Minas Gerais. A pesquisa buscou identificar quais os fatores que influenciam a avaliação da qualidade do serviço prestado aos passageiros e, para tal, foram avaliadas as características valorizadas pelos passageiros. O estudo tem natureza aplicada, exploratória e descritiva, classificando-se como um trabalho quantitativo, pois utilizou de modelos estatísticos para explicar os dados obtidos. Este estudo demonstrou que a adaptação da escala SERVQUAL, com as suas cinco dimensões originais apresentou quatros fatores, por ordem, que influenciaram a qualidade do serviço prestado: garantia e confiabilidade, tangibilidade, empatia e responsividade. $\mathrm{O}$ estudo proporcionará à organização estabelecer ações em seu planejamento estratégico considerando os resultados e o ambiente do novo cenário diante da pandemia.

Palavras-chave: Transporte rodoviário interestadual de passageiros; Escala SERVQUAL; Pandemia da COVID-19.

\begin{abstract}
This article presents an application of the SERVQUAL tool adapted to evaluate the service quality provided in an interstate passenger transport company in the state of Minas Gerais. The research sought to identify which factors influence the quality evaluation of service provided to passengers and, for this purpose, the characteristics valued by passengers were evaluated. The study has an applied, exploratory and descriptive nature, being classified as a quantitative work, as it used statistical models to explain the data obtained. This study demonstrated that the adaptation of SERVQUAL scale, with its five original dimensions, presented four factors, in sequence, which influence the quality service provided: assurance and reliability, tangibility, empathy and responsiveness. The study will enable the organization to establish actions in its strategic planning considering the results and the new scenario
\end{abstract} environment facing the pandemic.

Keywords: Interstate passenger transport system; SERVQUAL scale; COVID-19 pandemic.

\section{Resumen}

Este artículo presenta una puesta en práctica de la herramienta SERVQUAL adaptada para evaluar la calidad del servicio ofrecido en una empresa de transporte carretero interestatal de pasajeros en el estado de Minas Gerais. La investigación buscó identificar qué factores influyen en la evaluación de la calidad del servicio ofrecido a los pasajeros y, para ello, se evaluaron las características valoradas por los pasajeros. El estudio tiene un carácter aplicado, exploratorio y descriptivo, clasificándose como un trabajo cuantitativo, porque utilizó modelos estadísticos para explicar los datos obtenidos. Este estudio demostró que la adaptación de la escala SERVQUAL, con sus cinco dimensiones originales, presenta cuatro factores, en orden, que influyen en la calidad del servicio prestado: garantía y confiabilidad, tangibilidad, empatía y capacidad de respuesta. El estudio permitirá a la organización establecer acciones en su planificación estratégica considerando los resultados y el entorno del nuevo escenario frente la pandemia.

Palabras clave: Transporte carretero interestatal de pasajeros; Escala SERVQUAL; Pandemia de COVID-19. 


\section{Introdução}

A demanda por transportes aumenta devido as relações econômicas e sociais que os indivíduos desenvolvem entre si (Barabino \& Deiana, 2013). O transporte rodoviário de passageiros é um indutor que promove o deslocamento de pessoas, estimula o desenvolvimento do país ao conectar as pessoas aos seus diversos interesses. O transporte rodoviário de passageiros por ônibus no Brasil é o modo de condução mais utilizado pela população devido à regularidade dos serviços ofertados, abrangência da malha rodoviária e o valor das tarifas mais acessíveis se comparado ao modal aéreo (Freitas, Reis Filho, \& Rodrigues, 2011).

Neste contexto, saber se posicionar, compreender mais detalhadamente o mercado e conhecer o processo de escolha dos passageiros se tornou vital para as empresas que atuam neste competitivo mercado e que enfrentam problemas como: “queda na demanda transportada, elevação de custos, falta de políticas públicas, omissão governamental na regulação de situações que se desviam do modelo de competição perfeita” (Rolim \& Brasileiro, 2011, p. 15). Há que se considerar ainda a divisão do mercado com alguns concorrentes indiretos; empresas clandestinas, frota fretada, carros de passeio, empresas de aviação (Freitas, Reis Filho, \& Rodrigues, 2011).

A mobilidade urbana tem sido marcada pela ascensão de soluções inovadoras de transportes, os quais também favoreceram a entrada de novos concorrentes nesse meio, tais como as caronas compartilhadas, o uber e o buser, aplicativos que se popularizaram com a oferta de serviços de viagens sob demanda.

O Estado trouxe novos desafios para o meio, ao flexibilizar o acesso por meio da abertura de mercado, com uma política liberal, através do Decreto n 10.157/2019 (2019), ao editar as regras de desregulamentação do setor de transporte interestadual de passageiros, considerado um setor marcado tradicionalmente por monopólio.

Uma das características fundamentais à sobrevivência de qualquer organização é a capacidade de antecipar aos riscos diante sinalizados pelo mercado, neste processo, os principais fatos que impactaram são decorrentes de eventos imprevistos e outros não tão bem dimensionados como os novos entrantes no meio (uber, buser, caronas compartilhadas), concorrência de preços do meio aéreo, frotas clandestinos, carros próprios e a mudança de legislação promovida pelo governo, como dito anteriormente, surpreendeu-as e afetou-as significativamente.

Soma-se nesta análise de cenário, o surgimento da doença infecciosa Coronavirus Disease 2019 (COVID-19), impactando fortemente este nicho de mercado, devido a necessidade de restringir o movimento não essencial de pessoas com a finalidade de conter a propagação dos casos da doença. De acordo com o Centro Europeu de Prevenção e Controle de Doenças (https://europa.eu/european-union/about-eu/agencies/ecdc_pt), a epidemia surgiu em fins de 2019, e se espalhou pela Europa no início de 2020, chegando ao Brasil no mesmo período. Desde o dia 16 de março de 2020, momento em que as medidas de isolamento social foram sugeridas ou implementadas, observou-se a retração da demanda do transporte de passageiros de forma significativa no país. Desta forma, o setor de transportes vem convivendo com a redução da demanda transportada, diminuição da oferta de serviços (determinadas pelas orientações sanitárias), falência de empresas, demissões e suspensão de contratos dos trabalhadores, redução de jornadas de trabalho.

O objeto de estudo desta pesquisa foi o de investigar a percepção da qualidade na prestação dos serviços prestados em uma empresa de transporte rodoviário de passageiros interestadual, localizada na Zona da Mata Mineira nos tempos atuais. Para responder o problema de pesquisa foi escolhido o nicho do transporte rodoviário interestadual por ser a parte de maior faturamento da mesma e, portanto, suscetível de ações de curto prazo para minimizar as perdas ocorridas. O instrumento de pesquisa escolhido foi construído com as cinco dimensões da qualidade em serviços, por meio da adaptação da escala SERVQUAL (Parasuraman, Zeithaml, \& Berry, 1988).

Tendo como motivação conhecer as características valorizadas pelos passageiros diante do serviço prestado, o presente estudo tem enfoque na obtenção da importância dos atributos da qualidade no transporte rodoviário interestadual de 
passageiros fundamentais para o planejamento estratégico da organização em face do cenário traçado.

\section{Referencial Teórico}

Nesta seção são apresentadas as principais características do serviço de transporte de passageiros, o modelo adotado para análise de prestação de serviços (SERVQUAL) e os principais desafios enfrentados pelo transporte regular interestadual de passageiros.

\subsection{Dimensões da qualidade de serviços de transporte e sua aplicabilidade ao setor de transporte de passageiros}

Sob a ótica do cliente, a impressão mais imediata de serviço ocorre no momento que interage com a empresa, o "momento da verdade" e esse encontro, pode desempenhar um papel determinante da satisfação de um cliente com a empresa (Bitner, Booms, \& Mohr, 1994).

Zeithaml et al. (1985) realizaram uma revisão da literatura e encontraram 46 publicações de 33 estudos com base nas características dos serviços, e reuniram as abordagens desses diversos autores em seu estudo denominado dimensões da qualidade de serviços prestados. Os autores observaram que as características, mais frequentemente citadas, foram intangibilidade, inseparabilidade, heterogeneidade e perecibilidade.

A atividade de transporte rodoviário de passageiros por ônibus é considerada tipicamente como prestação de serviço com algumas características próprias do meio (Freitas et al., 2011). Nesta atividade, o passageiro é visto como cliente do sistema e das empresas operadoras cuja expectativa no momento da compra é de que o serviço será bem executado, proporcionando-lhe satisfação e motivando-o na continuidade da utilização do sistema de transporte público (Ferraz \& Torres, 2004).

Entre os atributos presentes no meio setor de transporte de passageiros destacam-se as características presentes no Quadro 1:

Quadro 1: Características dos serviços de transportes.

\begin{tabular}{|c|c|}
\hline Intangibilidade & $\begin{array}{l}\text { Marcada pelas experiências da interação do usuário com o serviço de transporte, sendo interpretadas à luz das } \\
\text { expectativas particulares de cada passageiro sobre o serviço prestado (Bertozzi \& Lima Junior, 1998). }\end{array}$ \\
\hline Inseparabilidade & $\begin{array}{c}\text { Na maioria das vezes o pagamento é realizado antes da prestação do serviço, o que geralmente não possibilita o } \\
\text { usuário desistir do serviço (Freitas et al., 2011, p. 51). }\end{array}$ \\
\hline Heterogeneidade & $\begin{array}{l}\text { Cada viagem pode ser considerada como um serviço específico (heterogeneidade), pois é influenciada por } \\
\text { diversos aspectos (condições climáticas, condições de tráfego, condições do veículo, quantidade de passageiros, } \\
\text { etc.), tornando difícil ou quase impossível a detecção e correção de falhas e problemas antes que eles ocorram e } \\
\qquad \text { afetem o usuário (Freitas et al., 2011, p. 51). } \\
\text { O serviço de transporte por ônibus é prestado e consumido de forma coletiva, em que os usuários nem sempre } \\
\text { apresentam o mesmo perfil e nem compartilham o mesmo pensamento (Freitas et al., 2011, p. 51). } \\
\text { A prestação do serviço é muito dispersa espacialmente e temporalmente, dificultando a padronização das } \\
\text { atividades, supervisão e o controle de pessoal (Freitas et al., 2011, p. 51). }\end{array}$ \\
\hline Perecibilidade & $\begin{array}{l}\text { O serviço de transporte não pode ser armazenado - se os passageiros não embarcam em um horário, nem sempre } \\
\text { é possível acomodá-los em outro horário e o serviço não é prestado na totalidade (Freitas et al., 2011, p. 51). } \\
\text { Os assentos não ocupados nos ônibus não podem ser recuperados (Zeithaml et al., 1985). }\end{array}$ \\
\hline Tangibilidade & $\begin{array}{l}\text { O transporte rodoviário de passageiros apresenta uma parcela de aspectos tangíveis que são; as características } \\
\text { dos veículos e dos pontos de venda de passagem, pessoal de operação e clientes. (Ferraz \& Torres, 2004) }\end{array}$ \\
\hline
\end{tabular}


No caso da atividade de transportes, há predominância do uso de elementos tanto tangíveis quanto intangíveis. Dessa maneira, embora um serviço compreenda uma parcela de elementos tangíveis, como é o caso do transporte rodoviário de passageiros, cabe ao passageiro apenas usufruir do serviço. E dessa forma, o passageiro pode, por exemplo, tangibilizar o serviço, compartilhando sua experiência por meio das redes sociais, ou comunicação boca-a-boca, que podem ter os aspectos de satisfação/insatisfação expressos como reclamações, elogios, sugestões, reivindicações diante da percepção do serviço prestado a ele.

Dadas as condições que permeiam o sistema de transporte rodoviário de passageiros, é um desafio para os prestadores de serviço gerenciar a relação com os clientes, de maneira que o atendimento entregue proporcione uma percepção satisfatória e estimule a continuidade da utilização do sistema de transporte público, pois "passageiro (cliente) não satisfeito, em curto, médio ou longo prazo, buscará outra opção de transporte" (Ferraz \& Torres, 2004, p. 99).

\subsection{Qualidade em serviços - SERVQUAL: um modelo de avaliação da qualidade de serviços}

Estudos em torno da qualidade em serviços têm sido objeto de pesquisa há décadas e considerados um fator de sucesso na competição entre empresas (Ladhari, 2009). Devido às características particulares presentes no meio, definir qualidade em serviço é uma tarefa complexa, difusa e abstrata (Sánches-Pérez, Abad, Carrillo, \& Fernández, 2007). Na visão de Parasuraman et al. (1988) e Grönroos (2003) a qualidade percebida em serviço é definida como a diferença entre as percepções e as expectativas dos clientes. $\mathrm{E}$ as expectativas que os clientes criam promovem um entendimento subjetivo em torno da qualidade do serviço (Sánches-Pérez et al., 2007).

Diante de um mercado cada vez mais competitivo, as necessidades das pessoas e a maneira como a sociedade encara a prestação de serviços mudam, ou seja, há maiores níveis de exigência, dessa forma, um dos desafios enfrentados pelas organizações prestadoras de serviço é descobrir os anseios desse cliente e a qualidade se revela como uma aliada para a sobrevivência das empresas frente a esta demanda.

A escala SERVQUAL é conhecida como a mais tradicional ferramenta destinada a mensuração da qualidade percebida em serviços (Hughey, Chawla, \& Khan, 2003). A ferramenta apresenta como vantagem a possibilidade de adaptação e ampliação para se adequar a realidade da organização que a utilizará (Parasuraman et al., 1988). Observa-se o crescimento contínuo da utilização da metodologia SERVQUAL no campo acadêmico e ambiente empresarial (Philip \& Hazlett, 1997). A ferramenta tem sido adaptada e empregada em áreas como educação, serviços hospitalares, bancos e apesar de um número limitado de estudos, algumas pesquisas têm adotado a escala SERVQUAL modificada para explorar a área de transporte público de passageiros (Barabino, Deiana, \& Tilocca, 2012).

Partindo da premissa que o usuário tem a capacidade de definir e influenciar a qualidade Parasuraman et al. (1988) criaram e aprimoraram a ferramenta. A escala é composta por 22 itens, alocados dentro das cinco dimensões da qualidade, com uma escala do tipo likert de sete pontos variando de "Concordo Totalmente (7)" para "Discordo Totalmente (1)". O instrumento é dividido em duas partes; a primeira as afirmações relacionadas às "expectativas" (E) e, em seguida, as declarações correspondentes a "percepções” (P) que formam a segunda parte, conforme Quadro 2.

Quadro 2: Características dos serviços de transportes.

\begin{tabular}{|c|c|}
\hline Dimensão & Características \\
\hline Tangibilidade & Instalações físicas, equipamentos e aparência do pessoal. \\
\hline Confiabilidade & Capacidade para realizar o serviço prometido de maneira confiável e com precisão. \\
\hline Responsividade & Aptidão para ajudar os clientes e fornecer pronto atendimento. \\
\hline Garantia & Conhecimento e cordialidade dos funcionários e sua capacidade de inspirar confiança e segurança. \\
\hline Empatia & Cuidado, atenção individualizada que a empresa oferece clientes. \\
\hline
\end{tabular}


Fonte: Parasuraman et al. (1988, p. 23).

A aplicabilidade ao setor pesquisado se explica na medida em que os usuários de ônibus possuem necessidades diversas como chegar ao trabalho, estudar, ir ao médico, visitar parentes e amigos, retornar para casa, adicionando a este serviço a qualidade que se descreve na conformidade do transporte à sua necessidade, e, considerando a avaliação dos atributos de conforto, segurança, acessibilidade, regularidade, tempo de viagem, confiabilidade, características dos veículos, sistemas de informação, comportamento dos operadores, conectividade, características dos locais de parada (Ferraz \& Torres, 2004).

Conforme o Relatório 100 do Transit Cooperative Research Program (Transportation Research Board \& Kittelson and Associates, Inc., 2003) a qualidade em transportes é definida pela performance total medida ou percebida do serviço prestado sob a ótica do passageiro. E, de acordo com esse relatório, os indicadores de frequência, horas de serviço, cobertura do serviço, demanda de passageiros, confiabilidade do serviço, diferença de tempos de viagem entre o automóvel e ônibus são alguns dos medidores para avaliar a qualidade em transporte são importantes atributos de mensuração.

\subsection{Os desafios no transporte regular interestadual de passageiros}

Com as mudanças ocorridas na legislação inerente ao transporte rodoviário interestadual de passageiros no Brasil, advindas do Decreto $\mathrm{n}^{\mathrm{o}} 10.157 / 2019$, que dispõe sobre a "Política Federal de Estímulo ao Transporte Rodoviário Coletivo Interestadual e Internacional de Passageiros” (2019), tratou da liberdade de preços, itinerário e de frequência, livre concorrência e redução do custo regulatório. Considerado como uma política de livre mercado, linhas que eram exploradas exclusivamente por determinadas empresas, agora poderão dividir as suas linhas com novos operadores.

As mudanças ocorreram com a intenção de aumentar a concorrência e com isso promover queda no preço da passagem e aumentar a qualidade do serviço prestado, portanto, são medidas promovidas para fazer frente a uma estrutura de operações com indícios de cartelização e "jogos arranjados" no desenho que amalha de linhas assumiu ao longo de décadas (Bazani, 2019). As empresas de transporte rodoviário de passageiros atuam no mercado com vários concorrentes, podendo variar de região para região, de acordo com o Quadro 3, pode-se elencar.

Quadro 3: Tipos de transporte.

\begin{tabular}{|c|c|}
\hline $\begin{array}{l}\text { Transporte } \\
\text { clandestinos }\end{array}$ & $\begin{array}{l}\text { Nomeia-se "clandestino" o "transporte remunerado de pessoas sem concessão, autorização ou permissão do Poder } \\
\text { Público competente" (Confederação Nacional do Transporte [CNT], 2017, p. 27). }\end{array}$ \\
\hline Frota Fretada & $\begin{array}{l}\text { "É composta por ônibus, micro-ônibus e automóveis fretados por empresas ou por grupos de interesse" (Freitas } e t \\
\qquad a l ., 2011, \text { p. 49). }\end{array}$ \\
\hline Carros de passeio & $\begin{array}{l}\text { "Que deixaram de ser restritos a uma pequena classe, passando a atender um grande contingente de brasileiros, por } \\
\text { meio da facilidade de pagamento" (Freitas et al., 2011, p. 50). }\end{array}$ \\
\hline $\begin{array}{l}\text { Empresas de } \\
\text { aviação }\end{array}$ & $\begin{array}{l}\text { "Que procuram, mais efetivamente, socializar seus serviços através de preços mais baixos e melhores formas de } \\
\text { pagamento" (Freitas et al., 2011, p. 50). Um estudo realizado por Turolla, Vassallo e Oliveira (2008) revelou que as } \\
\text { passagens rodoviárias são afetadas pelo preço das passagens aéreas, evidenciando a existência de uma relação entre } \\
\text { essas modalidades de transporte e também um potencial efeito substitutivo. Tal fato foi relatado pela Agência } \\
\text { Nacional de Aviação Civil (ANAC), que evidenciou a queda de passageiros transportados por ônibus interestadual, } \\
\text { desde o ano de } 2010 \text { e consequentemente o aumento pela demanda área (ANAC, 2020). }\end{array}$ \\
\hline
\end{tabular}

Fonte: Elaborado pelos autores (2020). 
A transformação digital também trouxe novos entrantes para o mercado diante da necessidade de adaptação das empresas de transporte rodoviário aos desafios da tecnologia, a partir da implantação de vendas on-line e o autoatendimento, conforme Quadro 4.

Quadro 4: Tipos de transporte de concorrência.

\begin{tabular}{|c|c|}
\hline Uber & $\begin{array}{r}\text { Conhecido como um prestador de serviços eletrônicos na área do transporte privado, através de um aplicativo que } \\
\text { permite a busca por motoristas baseada na localização. }\end{array}$ \\
\hline Buser & $\begin{array}{r}\text { Conhecido como “Uber do Ônibus" é um serviço de demanda. A empresa, com origem em Minas Gerais, se caracteriza } \\
\text { como fretamento coletivo, pelo qual, diferentes pessoas, pelo aplicativo, fretam viagens que vão surgindo de acordo } \\
\text { com a procura (Bazani, 2019). }\end{array}$ \\
\hline Aplicativos & $\begin{array}{r}\text { Aplicativos de caronas compartilhadas como "Blá, blá, blá Car”, dentre outros aplicativos, com finalidade de conectar } \\
\text { pessoas com destinos comuns, com preços atrativos e sem baldeações. }\end{array}$ \\
\hline
\end{tabular}

Fonte: Elaborado pelos autores (2020).

Os dados do Anuário de Transportes 2018, evidenciam a queda de passageiros no transporte rodoviário interestadual com trechos acima de $75 \mathrm{~km}$, de 2016 para a 2017 a queda foi de $7 \%$, de 2015 para 2016 queda de $12 \%$ e 2014 para 2015 queda de $8 \%$ (CNT, 2019).

Em meados do ano de 2020 surgiu a COVID-19, doença infecciosa Coronavirus Disease 2019, classificada como uma pandemia global, atingindo a mobilidade de pessoas e cargas em todo o mundo e vem impactando diretamente os comportamentos e estilos de vidas dos seres humanos, incluindo o modo de trabalho, consumo e viagens além da circulação de pessoas. As medidas de isolamento social exigiram um preço elevado da economia do país, e neste contexto, o transporte está entre os setores e segmentos mais afetados por essa crise (Lima, Schechtman, Brizon, \& Figueiredo, 2020).

Países fecharam fronteiras, cidades brasileiras restringiram a expedição de ônibus de viagens interestaduais nas rodoviárias, e, com o fechamento das divisas, houve, como resultado, uma intensa redução na demanda de transporte não somente em nível local, mas estendendo às esferas regionais e continentais. Outro fato emergido com a pandemia foi a implementação mais intensa do trabalho remoto (home office), tal acontecimento pode ser considerado o maior ensaio global no tocante à gestão da demanda de transportes (Ibold, Medimorec, \& Wagner, 2020).

Diante da conjuntura exposta, tem-se que a manutenção dos serviços de transporte de passageiros se defronta com desafios do nicho de atuação (decorrentes do uber, buser, caronas compartilhadas) de políticas públicas (abertura de mercado), mas também decorrentes do ambiente externo após a flexibilização do isolamento social. Neste cenário, considera-se que os operadores, sociedade e o poder público, busquem alternativas para a sobrevivência e continuidade do sistema (Lima et al., 2020).

\section{Metodologia}

Este estudo, trata-se de um estudo de caso, concentrado na avaliação do serviço prestado, sob a ótica do passageiro, de uma empresa da Zona da Mata Mineira, que dispõe de linhas conectando algumas cidades do estado de Minas Gerais ao estado do Rio de Janeiro.

A pesquisa tem uma abordagem quantitativa devido ao modelo escolhido para análise dos dados. Os dados foram tratados, primeiramente, por meio de estatística descritiva seguida da análise fatorial, procedimento estatístico multivariado, que busca "analisar a estrutura das interrelações (correlações) entre um grande número de variáveis [...], definindo um conjunto de dimensões latentes comuns, chamadas de fatores” (Hair Jr., Babin, Money, \& Samouel, 2005, p. 91). A análise fatorial pode ser exploratória ou confirmatória, este trabalho utilizou-se da primeira, pois tem como objetivo explorar os dados a fim de 
obter informações sobre o número de fatores necessários para melhor retratar os dados (Hair Jr. et al., 2005). Foi utilizado o software de análise estatística SPSS (IBM SPSS Statistics Versão 22®) para realizar as análises estatísticas.

Para a realização da análise fatorial exploratória, foram seguidos os passos sugeridos por Fávero, Belfiore, Silva e Chan (2009) e Malhotra (2012) que são: a matriz de correlação constituída pelas estatísticas Kaiser-Meyer-Olkin (KMO) e o teste de esfericidade de Bartlett; a extração de fatores iniciais, que pode ser realizado pela análise de componentes principais; a definição da quantidade de fatores seguidos pela rotação e da interpretação dos mesmos pelo método Varimax. Uma vez identificados os fatores, o passo seguinte foi examinar a confiabilidade com relação às suas variáveis originais, para isso foi utilizado coeficiente alfa, ou o Alfa de Cronbach $(\alpha)$.

Da escolha do modelo a investigações empírica, teve como objetivo investigar as particularidades de grupos de indivíduos examinados por meio de variáveis quantificadas nos dados coletados (Marconi \& Lakatos, 2002). A seleção dessas linhas é de cunho estratégico e econômico, e por terem sido afetados com a queda de passageiros.

Com relação aos objetivos, esta pesquisa classifica-se como exploratória e descritiva. O caráter exploratório resulta do fato de que a maioria das pesquisas existentes se refere ao transporte público por ônibus urbanos e também da inviabilidade de transportar resultados de estudos realizados no exterior devido à diferença entre as realidades presentes (Freitas et al., 2011). O traço descritivo está presente pois o trabalho procura retratar as características de uma população específica (Gil, 2008).

O instrumento usado foi um questionário, dividido em três etapas. A primeira parte constituída por vinte e duas questões tendo como com base na escala SERVQUAL (Parasuraman et al., 1988; Santos, 2014) adaptada à realidade investigada e às necessidades do setor, com uma escala tipo Likert com sete pontos. A segunda e terceira parte destinada a limitar o público-alvo da pesquisa, caracterizando dados pessoais e seleções como: gênero, idade, escolaridade, estado civil, renda familiar, motivo e frequência de viagens, local de compra, meios alternativos de viagem (Azevedo, Castro, \& Sampaio, 2020).

Portanto, o trabalho é uma pesquisa aplicada, com a intenção de gerar entendimento para gestão organizacional e orientado à resolução de questões específicas (Silva \& Menezes, 2005). O estudo possui um corte transversal, visto que, os dados foram coletados em uma única vez. A pesquisa foi realizada no período do primeiro semestre de 2020, sendo aplicados um total de 340 questionários. Do montante aplicado foram selecionados 225 pertinentes a análise e, portanto, válidos para a pesquisa, 115 questionários que não apresentavam todas as respostas ou respondidos de maneira incompleta foram desconsiderados. A seguir, as análises dos dados obtidos e as conclusões da pesquisa.

\section{Resultados e Discussão}

Esta pesquisa é resultado da dissertação de mestrado da primeira autora, cujos resultados possibilitaram a publicação de mais de um artigo, sendo que outras informações podem ser acessadas em Azevedo et al. (2020). Encontra-se nesta seção o tratamento estatístico dado à amostra e os métodos utilizados para sua interpretação.

Dos 225 respondentes, 130 (57,78\%) eram mulheres e 95 foram respondidos por homens (42,22\%). No quesito idade, tem-se a maioria dos indivíduos entre 16 a 25 anos, com 30,7\% da amostra, seguido pelo grupo de 26 a 35 anos, com 27,1\%. Em relação ao nível de escolaridade, tem-se como maioria o "ensino médio" representando 36,89\%, seguido em segundo lugar pelo "ensino superior" com 27,56\%. Com relação ao estado civil, a quantidade de solteiros (as) sobressaiu às demais, tendo $56 \%$ de "solteiros (as)" e 34,22\% de "casados (as)". Considera-se 32,89\%, possuem renda familiar acima de R $\$ 4.124,00$. A segunda faixa mais declarada está entre $\mathrm{R} \$ 2.063$ a $\mathrm{R} \$ 3.093,00$, com 24,89\%.

Quanto ao motivo da viagem declarado, destaca-se de forma isolada "visitas parentes/amigos" com $43,11 \%$ seguido por "lazer/turismo" com 29,78\%, percebe-se que ambas as categorias somadas equivalem 72,89\% da amostra. Com relação à frequência de viagens, nota-se que o uso de forma "eventual" é primordial sobre as demais frequências, com 51,56\% seguida pela frequência mensal com $21,33 \%$, contatou-se que a maioria dos entrevistados $(70,22 \%)$ utiliza como meio alternativo o 
carro. Quanto ao local de compra de passagens, prevalece o ponto de vendas físico com 57,78\%, que pode estar situado no interior de uma rodoviária ou ponto de parada de ônibus. O segundo meio mais utilizado com 35,56\% é a internet.

As variáveis constituídas por cada pergunta do questionário foram inseridas no software SPSS para realizar a primeira fase da análise fatorial exploratória para a redução de dimensão através do fator. Foi verificado que os dados apresentavam correlações aceitáveis para tornar adequada a análise fatorial, pois o KMO é de 0,880, que de acordo com Fávero et al (2009) é considerado uma boa medida. O teste de Bartlett foi bastante significativo (Sig. =0,000), o que indica a rejeição da hipótese nula $\left(\mathrm{H}_{0}\right)$, ao nível de significância de $5 \%$, $\left(\mathrm{H}_{0}\right.$ : matriz de correlações $\boldsymbol{\rho}$ seja estatisticamente igual à matriz identidade $\mathbf{I}$ de mesma dimensão) (Hair Junior et al, 2005). Nesse sentido, pode-se concluir que a aplicação da análise fatorial é apropriada, conforme Quadro 5.

Quadro 5: Teste KMO e Bartlett

\begin{tabular}{|l|c|c|}
\hline \multicolumn{2}{|c|}{ Medida Kaiser-Meyer-Olkin de adequação de amostragem } & 0,88 \\
\hline Teste de esfericidade de Bartlett & Aprox. Qui-quadrado & 2462,176 \\
\cline { 2 - 3 } & df & 231 \\
\cline { 2 - 3 } & Sig & 0 \\
\hline
\end{tabular}

Fonte: Elaborado pelos autores (2020).

A Tabela 1 analisou a comunalidade, que identifica a variância das variáveis alocadas distribuída com a solução, tendo como base apenas cargas acima de 0,50, tem-se que valores abaixo do estipulado poderão ser excluídos, uma vez que, tais variáveis não explicariam os fatores (Hair Jr. et al., 2005).

Tabela 1: Comunalidade.

\begin{tabular}{|c|c|c|}
\hline Variável & Inicial & Extração \\
\hline VT1 & 1,000 & 607 \\
\hline VT2 & 1,000 &, 732 \\
\hline VT3 & 1,000 & ,462 \\
\hline VT4 & 1,000 & 696 \\
\hline VT5 & 1,000 &, 515 \\
\hline VC6 & 1,000 & ,588 \\
\hline VC7 & 1,000 & ,681 \\
\hline VC8 & 1,000 & ,619 \\
\hline VC9 & 1,000 & 627 \\
\hline VP10 & 1,000 & 607 \\
\hline VP11 & 1,000 & 601 \\
\hline VP12 & 1,000 & ,446 \\
\hline VP13 & 1,000 &, 321 \\
\hline VS14 & 1,000 &, 716 \\
\hline VS15 & 1,000 & 682 \\
\hline VS16 & 1,000 & ,619 \\
\hline VS17 & 1,000 &, 524 \\
\hline VE18 & 1,000 &, 581 \\
\hline VE19 & 1,000 &, 742 \\
\hline VE20 & 1,000 & ,619 \\
\hline VE21 & 1,000 & 635 \\
\hline VE22 & 1,000 & ,392 \\
\hline
\end{tabular}


Os valores mais próximos de zero significam que os fatores comuns explicam pouca ou nenhuma variância comum dentro da variável, e "quanto mais próximas as comunalidades estão de 1, melhor os fatores explicarão os dados originais" (Field, 2009, p. 564). Com exceção de quatro variáveis que apresentaram valores abaixo de 0,5: "VT3" com 0,462, "VP12" com 0,446, "VP13" com 0,321 e "VE22" com 0,392, as quais foram retiradas da amostra, foi realizada novamente a extração de componentes principais. Assim, nesta segunda etapa, todas as variáveis que permaneceram apresentaram valores de comunalidade acima de 0,533 , e das 22 variáveis iniciais a quantidade foi reduzida para 18, conforme Tabela 2.

Tabela 2: Comunalidade segunda etapa.

\begin{tabular}{|c|c|c|}
\hline Variável & Inicial & Extração \\
\hline VT1 & 1,000 & ,683 \\
\hline VT2 & 1,000 & ,754 \\
\hline VT4 & 1,000 &, 735 \\
\hline VT5 & 1,000 &, 533 \\
\hline VC6 & 1,000 & ,611 \\
\hline VC7 & 1,000 & ,678 \\
\hline VC8 & 1,000 & ,655 \\
\hline VC9 & 1,000 & ,652 \\
\hline VP10 & 1,000 & ,634 \\
\hline VP11 & 1,000 & ,644 \\
\hline VS14 & 1,000 &, 725 \\
\hline VS15 & 1,000 & ,696 \\
\hline VS16 & 1,000 & ,600 \\
\hline VS17 & 1,000 &, 544 \\
\hline VE18 & 1,000 & ,616 \\
\hline VE19 & 1,000 & ,716 \\
\hline VE20 & 1,000 & ,661 \\
\hline VE21 & 1,000 & 663 \\
\hline
\end{tabular}

Fonte: Elaborada pelos autores (2020).

Após a retirada de quatro variáveis pelo critério da comunalidade, o KMO foi modificado de 0,880 para 0,879, permanecendo como um valor considerado "bom" para prosseguir com a análise fatorial. E o teste de Bartlett permaneceu com valor abaixo de 0,05 , evidenciando assim, que existem correlações significativas entre as variáveis.

Posteriormente, foi avaliado o número de fatores retidos, adotando como critério do autovalor acima de 1, no scree plot, Gráfico 1, o autovalor cujo valor é acima de 1 satisfaz a regra da raiz latente que são considerados significativos (Hair Jr. et al., 2005) e na verificação de interpretabilidade dos fatores, dessa forma encontrou-se quatro fatores retidos, representando 65,56\% da variabilidade dos dados (Tabela 3). De acordo com Malhotra (2012), recomenda-se que esse valor seja acima de $60 \%$. 
Research, Society and Development, v. 10, n. 7, e32510716538, 2021

(CC BY 4.0) | ISSN 2525-3409 | DOI: http://dx.doi.org/10.33448/rsd-v10i7.16538

Gráfico 1: Scree plot.

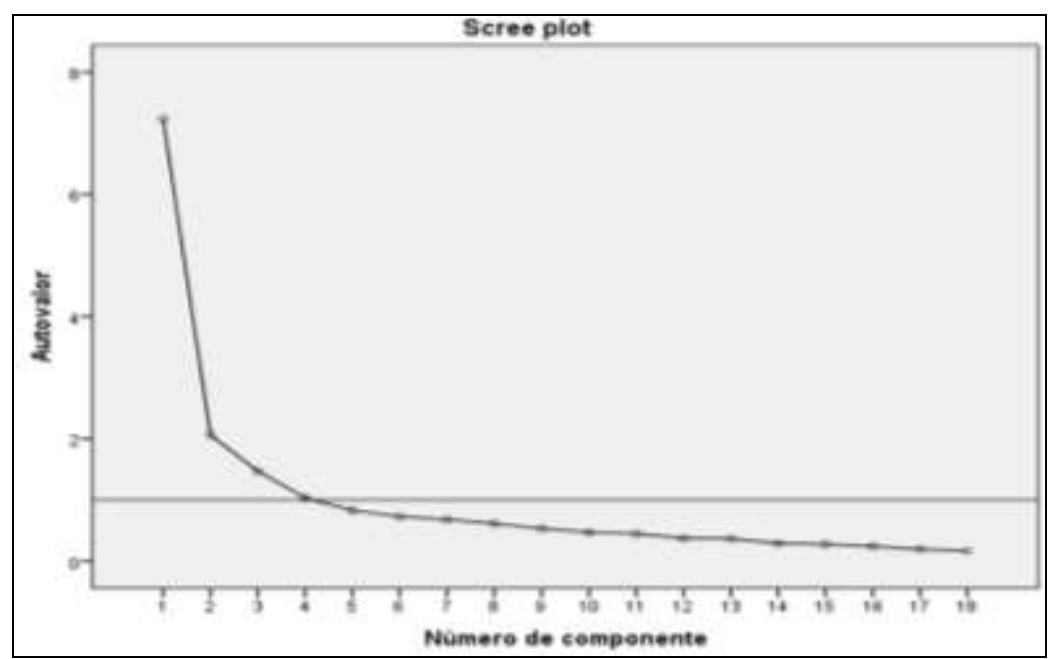

Fonte: Elaborado pelos autores (2020).

Tabela 3: Variância total explicada.

\begin{tabular}{|c|c|c|c|c|c|c|c|c|c|}
\hline \multirow[b]{2}{*}{ Var. } & \multicolumn{3}{|c|}{ Valores próprios iniciais } & \multicolumn{3}{|c|}{$\begin{array}{l}\text { Somas de extração de carregamentos ao } \\
\text { quadrado }\end{array}$} & \multicolumn{3}{|c|}{$\begin{array}{l}\text { Somas rotativas de carregamentos ao } \\
\text { quadrado }\end{array}$} \\
\hline & Total & $\begin{array}{c}\% \text { de } \\
\text { variância }\end{array}$ & $\%$ cumulativa & Total & $\begin{array}{c}\text { \% de } \\
\text { variância }\end{array}$ & $\%$ cumulativa & Total & $\begin{array}{c}\text { \% de } \\
\text { variância }\end{array}$ & $\%$ cumulativa \\
\hline 1 & 7,236 & 40,199 & 40,199 & 7,236 & 40,199 & 40,199 & 3,924 & 21,8 & 21,8 \\
\hline 2 & 2,061 & 11,447 & 51,647 & 2,061 & 11,447 & 51,647 & 3,528 & 19,599 & 41,399 \\
\hline 3 & 1,472 & 8,178 & 59,825 & 1,472 & 8,178 & 59,825 & 2,848 & 15,824 & 57,223 \\
\hline 4 & 1,033 & 5,74 & 65,565 & 1,033 & 5,74 & 65,565 & 1,502 & 8,343 & 65,565 \\
\hline 5 & 0,829 & 4,603 & 70,168 & & & & & & \\
\hline 6 & 0,728 & 4,044 & 74,212 & & & & & & \\
\hline 7 & 0,681 & 3,784 & 77,996 & & & & & & \\
\hline 8 & 0,613 & 3,403 & 81,399 & & & & & & \\
\hline 9 & 0,531 & 2,952 & 84,351 & & & & & & \\
\hline 10 & 0,47 & 2,612 & 86,963 & & & & & & \\
\hline 11 & 0,446 & 2,477 & 89,439 & & & & & & \\
\hline 12 & 0,373 & 2,072 & 91,511 & & & & & & \\
\hline 13 & 0,366 & 2,032 & 93,543 & & & & & & \\
\hline 14 & 0,289 & 1,607 & 95,15 & & & & & & \\
\hline 15 & 0,273 & 1,519 & 96,669 & & & & & & \\
\hline 16 & 0,242 & 1,344 & 98,013 & & & & & & \\
\hline 17 & 0,193 & 1,07 & 99,083 & & & & & & \\
\hline 18 & 0,165 & 0,917 & 100 & & & & & & \\
\hline
\end{tabular}

Fonte: Elaborada pelos autores (2020). 
O passo seguinte foi analisar a rotação, pelo método varimax, tendo como critério apenas cargas acima de 0,40, dessa forma, as cargas de fatores menores do que 0,40 não foram evidenciadas e as variáveis estão listadas por ordem de tamanho em seus respectivos fatores, na Tabela 4.

Tabela 4: Matriz de componente rotativa.

\begin{tabular}{|c|c|c|c|c|}
\hline \multirow{2}{*}{ Variável } & \multicolumn{4}{|c|}{ Componente } \\
\hline & 1 & 2 & 3 & 4 \\
\hline VS14 & 0,815 & & & \\
\hline VS15 & 0,778 & & & \\
\hline VS16 & 0,710 & & & \\
\hline VS17 & 0,610 & & & \\
\hline VT4 & & 0,821 & & \\
\hline VT2 & & 0,795 & & \\
\hline VT1 & & 0,757 & & \\
\hline VT5 & & 0,663 & & \\
\hline VC9 & 0,553 & 0,567 & & \\
\hline VC6 & 0,502 & 0,499 & & \\
\hline $\mathrm{VC} 8$ & 0,622 & 0,464 & & \\
\hline VC7 & 0,696 & 0,411 & & \\
\hline VE19 & & & 0,795 & \\
\hline VE20 & & & 0,771 & \\
\hline VE21 & & & 0,747 & \\
\hline VE18 & & & 0,700 & \\
\hline VP10 & & & & 0,757 \\
\hline VP11 & & & & 0,736 \\
\hline
\end{tabular}

Fonte: Elaborada pelos autores (2020).

Verifica-se que as variáveis "VC9", "VC6", "VC8" e "VC7" estão dispostas em dois fatores simultaneamente. "Quando uma variável demonstra ter mais de uma carga significante, ela é chamada de carga cruzada" (Hair Junior et al, 2009, p. 121).

A variável "VC9" corresponde ao quesito "Quando a Empresa promete fazer algo em certo tempo, realmente o faz?", "VC6" corresponde ao item “Quando você tem algum problema com a Empresa, ela é solidária e o deixa segura?", a variável "VC8" corresponde à questão "As viagens são cumpridas nos horários?" e a variável "VC7" que significa "A Empresa é de confiança?". Todas as quatro variáveis pertencem à dimensão da confiabilidade e estão alocadas ao mesmo tempo no fator 1 e 2. As variáveis que estão alocadas no fator 1 e que não possuem carga cruzada condizem com a dimensão garantia enquanto as variáveis em mesmas condições no fator 2 dizem respeito à dimensão tangibilidade.

Em uma prévia análise, percebe-se que as quatro variáveis estão mais teoricamente relacionadas ao fator 1 do que com o fator 2, pois confiabilidade pode ser interpretada como confiança, credibilidade da empresa perante seus usuários. Com o intuito de comprovar a real alocação das variáveis e testar a confiabilidade dos fatores foi realizada a medida alfa de Cronbach, para que cada variável se associe a apenas um único fator.

Com suporte do alfa de Cronbach, as variáveis com cargas cruzadas "VC9", "VC6", "VC8" e "VC7" foram testadas para serem reposicionadas de acordo com a sua respectiva carga fatorial, conforme Tabela 5. Em um primeiro momento, foi observada a carga fatorial da variável estando presente no primeiro fator em que estava alocada. Em seguida, as variáveis 
foram retiradas do fator 1, e então, calculado o alfa, novamente. De maneira análoga, o mesmo procedimento foi realizado para o fator 2. O critério de decisão do fator a ser realocado ou mantido depende da carga fatorial mais alta ou significante, conforme sugere Hair Jr., Black, Babin, Anderson e Tatham (2009) assumindo o valor de 0,60 como uma medida aceitável numa pesquisa exploratória, e 0,70 considerado aceitável em pesquisas em geral.

Tabela 5: Alfa de Cronbach com as variáveis de carga cruzada.

\begin{tabular}{|c|c|c|c|c|c|c|}
\hline Variável & $\begin{array}{c}\text { Variáveis } \\
\text { alocadas em } \\
\text { ambos os fatores }\end{array}$ & $\begin{array}{l}\text { Alfa inicial do } \\
\text { fator } 1\end{array}$ & $\begin{array}{l}\text { Alfa do fator } 1 \text { sem as } \\
\text { variáveis VC9, VC6, } \\
\text { VC8 e VC } 7\end{array}$ & $\begin{array}{c}\text { Alfa inicial } \\
\text { do fator } 2\end{array}$ & $\begin{array}{c}\text { Alfa do fator } 2 \text { sem as } \\
\text { variáveis VC9, VC6, } \\
\text { VC8 e VC } 7\end{array}$ & $\begin{array}{l}\text { Posição de } \\
\text { realocação }\end{array}$ \\
\hline VC9 & 1 e 2 & \multirow{4}{*}{-} & \multirow{4}{*}{0,830} & \multirow{4}{*}{0,894} & \multirow{4}{*}{0,843} & Fator 1 \\
\hline VC6 & 1 e 2 & & & & & Fator 1 \\
\hline VC8 & 1 e 2 & & & & & Fator 1 \\
\hline VC7 & $1 \mathrm{e} 2$ & & & & & Fator 1 \\
\hline
\end{tabular}

Fonte: Elaborada pelos autores (2020).

Como as quatro variáveis "VC9", "VC6", "VC8" e "VC7" pertencem a mesma dimensão, "confiabilidade", optou-se em retirar todas de cada fator, e assim, analisar individualmente o valor obtido do alfa de Cronbach do fator 1 e 2 separadamente após a extração.

De acordo com as cargas fatoriais obtidas com o modelo de alfa de Cronbach, as quatro variáveis "VC9", "VC6", "VC8" e "VC7" ficam alocadas no fator 1, pois caso sejam retiradas as quatro variáveis desse fator, ele ficaria com um valor (alfa de Cronbach) menor do que o do fator 2. A Tabela 6 evidencia o posicionamento dos itens com cargas cruzadas.

Tabela 6: Posições finais das variáveis com carga cruzada

\begin{tabular}{ccccc}
\hline Fator & $\begin{array}{c}\text { Quantidade de variáveis } \\
\text { inicial }\end{array}$ & Quantidade de variáveis final & Alfa inicial & Alfa final recalculado \\
\hline 1 & 8 & 8 & 0,891 & 0,891 \\
2 & 8 & 4 & 0,894 & 0,843 \\
\hline
\end{tabular}

Fonte: Elaborada pelos autores (2020).

Assim, finalizada a verificação das cargas cruzadas, o passo seguinte foi calcular o alfa de Cronbach para descobrir o grau de consistência interna ou confiabilidade das variáveis em estudo de cada fator. A análise de confiabilidade foi composta por 18 variáveis distribuídas em 4 fatores de acordo com a sua carga. A Tabela 7 apresenta em ordem crescente o grau de confiabilidade por fator.

Tabela 7: Alfa de Cronbach.

\begin{tabular}{ccccc}
\hline Fator & $\begin{array}{c}\text { Quantidade de variáveis } \\
\text { inicial }\end{array}$ & Quantidade de variáveis final & Alfa inicial & Alfa final recalculado \\
\hline 1 & 8 & 8 & 0,891 & 0,891 \\
2 & 8 & 4 & 0,894 & 0,843 \\
3 & 4 & 4 & 0,841 & 0,841 \\
4 & 2 & 2 & 0,552 & 0,552 \\
\hline
\end{tabular}


Os resultados relatam que, apesar de todas as escalas serem consideradas exploratórias, a maioria delas possui um valor (alpha de Cronbach) acima de 0,70, somente o fator 4 apresentou um valor abaixo de 0,60, o qual é considerado como um limite inferior de aceitabilidade da medida de Cronbach.

$\mathrm{Na}$ análise fatorial, foram identificados quatro fatores que influenciaram a qualidade do serviço prestado no transporte interestadual de passageiros, sob a ótica do usuário. O fator com maior grau de confiabilidade foi denominado de "Garantia e Confiabilidade". O fator 1, congregou as variáveis da dimensão "Garantia" e da dimensão "Confiabilidade". Este fator engloba aspectos relacionados ao "conhecimento e cordialidade dos funcionários e sua capacidade de inspirar confiança, segurança e também a capacidade da empresa para realizar o serviço prometido de maneira confiável e com precisão" (Parasuraman et al., 1988, p. 23). Assim, percebe-se que os passageiros se preocupam com o tempo de viagem, ou seja, a pontualidade, intervalo entre veículos, cumprimento do itinerário, condução segura do motorista (Bertozzi \& Lima Junior, 1998; Transportation Research Board \& Kittelson and Associates, Inc., 2003; Ferraz \& Torres, 2004). Santos (2014) corrobora esta perspectiva, pois a dimensão confiança, seguido pelo item conforto, foram os mais expressivos em sua pesquisa e se relacionam com a dimensão tangibilidade.

O segundo fator extraído foi "Tangibilidade", que pode ser interpretado pelas "instalações físicas, equipamentos e aparência do pessoal" (Parasuraman et al., 1988, p. 23). Aspectos compreendidos por este fator no modal de transportes de passageiros são: limpeza dos ônibus e seu estado de conservação, conforto englobando os bancos, iluminação, temperatura, barulho, aparência dos ônibus e funcionários (Bertozzi \& Lima Junior, 1998; Ferraz \& Torres, 2004). Em tempos de pandemia, este fator tomou uma proporção particular sob as regras específicas e protocolos de segurança no transporte de pessoas e cargas.

O terceiro fator evidenciado na pesquisa foi "Empatia." Neste caso, destacam-se questões relacionadas ao "cuidado, atenção individualizada que a empresa oferece aos clientes." (Parasuraman et al., 1988, p. 23). As características desse fator englobam questões relacionadas às relações humanas, de cordialidade cujos agentes são o motorista, o agente de vendas e outras pessoas da empresa em fornecerem informações, com atenção e educação, visando tratar os passageiros com respeito (Bertozzi \& Lima Junior, 1998; Ferraz \& Torres, 2004).

O quarto e último fator denominado "Responsividade" refere-se a "aptidão para ajudar os clientes e fornecer pronto atendimento" (Parasuraman et al., 1988, p. 23). Apesar de o fator ter apresentado um Alfa de Cronbach considerado frágil pela literatura $(\alpha=0,552)$, optou-se por mantê-lo como resultado da pesquisa, visto que se trata de um estudo preliminar. Essa dimensão aborda a disponibilidade de substituição imediata quando um veículo quebra na estrada, atendimento ao usuário, disponibilização e divulgação dos itinerários e horários das linhas, relação entre usuários (Bertozzi \& Lima Junior, 1998; Ferraz \& Torres, 2004).

\section{Conclusão}

Este estudo apresentou um registro do sistema de transporte rodoviário de passageiros interestadual entre os estados de Minas Gerais e Rio de Janeiro e, em especial, um estudo de caso, devido a essa característica particular, de análise transversal, não existe a intenção de esgotar todas as particularidades ao estudo da opinião dos passageiros em relação à qualidade do serviço prestado.

Verifica-se que o problema de pesquisa foi respondido, considerando que foram identificados quatros fatores que influenciam na qualidade do serviço prestado em momentos de pandemia da COVID-19. Dessa forma, o estudo contribui à medida em que proporcionou à gestão um olhar crítico, identificando pontos fortes e fracos da organização e possibilitou levantar as ameaças e oportunidades pertinentes ao escopo estratégico necessário para o enfrentamento da questão nos tempos atuais e a perspectiva de melhorar a oferta do serviço. 
Durante o desenvolvimento do estudo foi possível reconhecer a existência de limitações promovidas pela pandemia da COVID-19. Como, à época da pesquisa, não existia vacina contra o novo vírus e nem remédios para a cura da doença, o isolamento social foi a única maneira encontrada para evitar que os casos se espalhassem cada vez mais. Diante deste cenário, atendendo às determinações governamentais, ocorreu a suspensão temporária do serviço de ônibus interestadual da empresa desde o dia 24 de março de 2020, assim, desde então, a organização teve uma perda de 100\% da demanda transportada que se tinha antes da pandemia no transporte interestadual de passageiros. O retorno da operação deu-se somente em 10 de junho de 2020 com apenas $12,5 \%$ da frota em funcionamento devido a falta de procura.

Empresas de transporte convivem com um novo paradigma, a COVID-19, promove a necessidade de se pensar nos atuais procedimento de operação, com alguns questionamentos, os quais ainda sem respostas como: A COVID-19 causará uma alteração geral no comportamento de locomoção dos indivíduos? Devido aos regulamentos de distanciamento físico, o surto da COVID-19 incentivará a população ao uso particular de automóveis e motos em detrimento ao transporte coletivo? Os novos serviços de mobilidade, como carona e o uber, serão mais utilizados que o transporte coletivo?

Pesquisas futuras devem ser realizadas no sentido de avaliar o comportamento dos consumidores no período pós pandemia identificando as mudanças que vão impactar a gestão do negócio. Um planejamento estratégico coerente deve conceber o estudo de cenários como forma de enfrentamento dos desafios do setor e para ser uma alternativa atraente perante aos usuários e não usuários, não basta ser somente útil ou necessária, será preciso ser desejada como empresa prestadora de serviços com qualidade, e com a devida segurança aos passageiros que os tempos atuais requerem.

\section{Referências}

Agência Nacional de Aviação Civil. (2020). Transporte Interestadual Regular de Passageiros - Aéreo e Rodoviário (Relatório/2020), Brasília, DF. https://www.anac.gov.br/assuntos/dados-e-estatisticas/mercado-de-transporte-aereo/transporte-interestadual-de-passageiros-aereo-e-rodoviario.

Azevedo, L.A.C., Castro, M.C.D., \& Sampaio, D.O. (2020). Aplicação do modelo dos cinco gaps em momentos de pandemia da COVID-19: O caso de uma empresa de transporte da Zona da Mata Mineira. Brazilian Journal of Development, 6 (11), 92984-93004. https:// doi:10.34117/bjdv6n11-635.

Barabino, B., \& Deiana, E. (2013). On the Attributes and Influencing Factors of End-users Quality Perceptions in Urban Transport: An Exploratory Analysis. Procedia - Social and Behavioral Sciences, 87, 18-30. https://www.sciencedirect.com/science/article/pii/S1877042813040366.

Barabino, B., Deiana, E., \& Tilocca, P. (2012). Measuring service quality in urban bus transport: a modified SERVQUAL approach. International Journal of Quality and Service Sciences, 4 (3), 238-252. https://sci-hub.tw/https://doi.org/10.1108/17566691211269567.

Bazani, A. (2019). ANTP dá autorização para cooperativa de transporte operar linhas interestaduais de ônibus. Diário do Transporte. https://diariodotransporte.com.br/2019/11/28/antt-da-autorizacao-para-cooperativa-de-transporte-operar-linhas-interestaduais-de-onibus/.

Bertozzi, P. P., \& Lima Junior, O. F. (1998). A qualidade no serviço de transporte público sob as óticas do usuário, do operador e do órgão gestor. Revista dos Transportes Públicos, 21 (81), 53-66. http://files.antp.org.br/2016/4/4/revista-completa-81.pdf.

Bitner, M. J., Booms, B. H., \& Mohr, L. A. (1994). Critical Service Encounters: The Employee's Viewpoint. Journal of Marketing, 58 (4), 95-104. https://www.jstor.org/stable/1251919?seq=1.

Confederação Nacional do Transporte. (2017). Transporte rodoviário de passageiros em regime de fretamento. Brasília. http://cms.cnt.org.br/Imagens\%20CNT/PDFs \%20CNT/Estudos\%20CNT/2017\%20CNT\%20Transporte\%20Rodovi\%C3\%A1rio\%20de\%20Passageiros\%20e m\%20Regime\%20de\%20Fretamento\%20060317\%20(1).pdf.

Confederação Nacional do Transporte. (2019). Anuário CNT do Transporte: estatísticas consolidadas. Brasília. https://anuariodotransporte.cnt.org.br/2019/Inicial.

Decreto $n^{\circ}$ 10.157, de 4 de dezembro de 2019 (2019). Institui a Política Federal de Estímulo ao Transporte Rodoviário Coletivo Interestadual e Internacional de Passageiros. Brasília. 2019. http://www.in.gov.br/en/web/dou/-/decreto-n-10.157-de-4-de-dezembro-de-2019-231562129.

Fávero, L. P. L., Belfiore, P. P., Silva, F. L., \& Chan, B. L. (2009). Análise de dados: modelagem multivariada para tomada de decisões. Rio de Janeiro: Elsevier.

Ferraz, A. C. P., \& Torres, I. G. E. (2004). Transporte público urbano (2a ed.). São Carlos: Rima.

Field, A. (2009). Descobrindo a estatística usando o SPSS [recurso eletrônico]. (2 ${ }^{\mathrm{a}}$ ed.). (L. Viali, Trad.). 2. ed. Porto Alegre: Artmed. (Obra original publicada em 2005)

https://edisciplinas.usp.br/pluginfile.php/4320753/mod_resource/content/1/2011_Field\%20\%28completo\%29Descobrindo\%20a\%20estat\%C3\%ADstica\%20c om\%20SPSS.pdf. 
Freitas, A. L. P., Reis Filho, C. A. C., \& Rodrigues, F. R. (2011). Avaliação da qualidade do transporte rodoviário intermunicipal de passageiros: uma abordagem exploratória. Transportes, 19 (3), 49-61. https://doi.org/10.14295/transportes.v19i3.376.

Gil, A. C. (2008). Métodos e técnicas de pesquisa social (6a ed.). São Paulo: Atlas.

Grönroos, C. (2003). Marketing: gerenciamento e serviços (2a ed.). Rio de Janeiro: Elsevier.

Hair Jr., J. F., Babin, B., Money, A. H., \& Samouel, P. (2005). Fundamentos de métodos de pesquisa em administração. (F. A. Costa, Trad.). Porto Alegre: Bookman.

Hair Jr., J. F., Black, W. C., Babin, B. J., Anderson, R. E., \& Tatham, R. L. (2009). Análise multivariada de dados (6ª ed.). (A. S. Sant'Anna, Trad.). Porto Alegre: Bookman. (Obra original publicada em 1979).

Hughey, D. W., Chawla, S. K., \& Khan, Z. U. (2003). Measuring the Quality of University Computer Labs Using Servqual: A Longitudinal Study. Quality Management Journal, 10 (3), 33-44. Recuperado em 04 abril, 2020, de https://sci-hub.tw/https://doi.org/10.1080/10686967.2003.11919071.

Ibold, S., Medimorec, M., \& Wagner, A. (2020). O surto de COVID-19 e suas implicações para o transporte público sustentável: algumas considerações. https://www.sutp.org/o-surto-de-covid-19-e-suas-implicacoes-para-o-transporte-publico-sustentavel-algumas-consideracoes/.

Transportation Research Board \& Kittelson and Associates, Incorporated (2003). Transit Capacity and Quality of Service Manual (Report 100, 2nd ed.). Washington: Transportation Research Board. http://onlinepubs.trb.org/onlinepubs/tcrp/docs/tcrp100/Part0.pdf.

Ladhari, R. (2009). A review of twenty years of SERVQUAL research. International Journal of Quality and Service Sciences, 1 (2), 172-198. https://scihub.tw/https://doi.org/10.1108/17566690910971445.

Lima, G. C. L. S., Schechtman, R., Brizon, L. C., \& Figueiredo, M. Z. (2020). Transporte público e COVID-19: o que pode ser feito?. Rio de Janeiro: Centro de Estudos em Regulação e Infraestrutura da Fundação Getúlio Vargas. https://ceri.fgv.br/sites/default/files/publicacoes/202004/covid_e_mobilidade_urbana_fgv_ceri.pdf.

Malhotra, N. K. (2012). Pesquisa de Marketing. Uma orientação aplicada (6a ed.). (L. B. Ribeiro \& M. Stefani, Trad.). Porto Alegre: Bookman.

Marconi, M. A., \& Lakatos, E. M. (2002). Técnicas de pesquisa (5a ed.). São Paulo: Atlas.

Parasuraman, A., Zeithaml, V. A., \& Berry, L. L. (1988). SERVQUAL. A multiple-item scale for measuring consumer perceptions of service quality. Journal of Retailing, 64 (1), 12-40. https://pdfs.semanticscholar.org/d26a/2423f00ca372b424a029ae22521299f00ede.pdf.

Philip, G., \& Hazlett, S. (1997). The measurement of service quality: a new P-C-P attributes model. International Journal of Quality \& Reliability Management, 14 (3), 260-286. https://doi.org/10.1108/02656719710165482.

Rolim, F., \& Brasileiro, A. (2009). Competitividade na regulamentação do transporte rodoviário de passageiros: uma análise à luz de um modelo de auditagem. Transportes, 17, (1), 14-26. https://www.revistatransportes.org.br/anpet/article/view/72/61.

Sánches-Pérez, M., Abad, J. C. G., Carrillo, G. M. M., \& Fernández, R. S. (2007). Effects of service quality dimensions on behavioural purchase intention: a study in public-sector transport. Marketing Service Quality, 17, (2), 134-151. https://doi.org/10.1108/09604520710735164.

Santos, R. G. (2014). Propostas para melhoria contínua da qualidade do transporte público coletivo do distrito federal utilizando a escala SERVQUAL. Dissertação de Mestrado, Faculdade de Tecnologia da Universidade de Brasília, Brasília, DF, Brasil. https://repositorio.unb.br/bitstream/10482/16346/1/2014_RodrigoGuimaraesSantos.pdf.

Silva, E. L., \& Menezes, E. M. (2005). Metodologia da pesquisa e elaboração de dissertação (4a ed. rev.). Florianópolis: UFSC. https://projetos.inf.ufsc.br/arquivos/Metodologia_de_pesquisa_e_elaboracao_de_teses_e_dissertacoes_4ed.pdf.

Turolla, F. A., Vassallo, M. D., \& Oliveira, A. V. M. (2008). Intermodal competition in the Brazilian interstate travel market. Revista de Análisis Económico, 23 (1), 21-33. https://papers.ssrn. com/sol3/papers.cfm?abstract_id=2796549.

Zeithaml, V. A., Berry, L. L., \& Parasuraman, A. (1985). Problems and Strategies in Services Marketing. Journal of Marketing, 49 (1), 33-46. https://www.researchgate.net/publication/329047152_Problems_and_Strategies_in_Services_Marketing. 